\title{
Lentiviral vector with a radiation-inducible promoter, carrying the ING4 gene, mediates radiosensitization controlled by radiotherapy in cervical cancer cells
}

\author{
TAO MA ${ }^{1}$, RUI GUO ${ }^{2}$, XI WANG $^{3}$, WEN-TONG SHEN $^{1}$, MIN ZHU $^{1}$, YE-NING JIN ${ }^{1}$ and HAO-PING XU ${ }^{1}$ \\ Departments of ${ }^{1}$ Radiation Oncology and ${ }^{2}$ Nuclear Medicine, Ruijin Hospital, \\ Shanghai Jiaotong University School of Medicine, Shanghai 200025, P.R. China; ${ }^{3}$ Department of \\ Neurology, Hackensack Meridian Health JFK Medical Center, Edison, NJ 08820, USA
}

Received March 3 2020; Accepted October 19, 2020

DOI: $10.3892 / \mathrm{ol} .2020 .12328$

\begin{abstract}
The presence of hypoxia in solid tumors is considered one of the major factors that contribute to radiation resistance. The aim of the present study was to establish a therapeutic system, which can be controlled by radiation itself, to enhance radiosensitivity. For this purpose, a lentiviral gene therapy vector containing the human inhibitor of growth 4 (ING4) and its upstream promoter, human early growth response factor-1 (EGR1), which possesses the radiation-inducible characteristics to activate the transcription of its downstream genes, was constructed. Downstream fluorescence proteins were investigated to ensure that the EGR1 promoter was induced by irradiation. Furthermore, ING4 open reading frame (ORF) expression was detected by western blotting. The cell cycle was analyzed by fluorescence-activated cell sorting analysis $48 \mathrm{~h}$ after the cells were exposed to X-rays ranging between 0 and 8 Gy. In cells stably and transiently transfected with reporter plasmids, the EGR1-driver gene was sensitive to ionizing irradiation. Furthermore, irradiation-induced ING4 gene expression was observed. The enhanced ING4 expression increased the number of cells in the $\mathrm{G}_{2} / \mathrm{M}$ phase and decreased the proportion of cells in the $G_{1} / S$ phase. Therefore, ING4 expression inhibited cell proliferation and was associated with less colonies being formed. Furthermore, ING4 suppressed hypoxia-inducible factor $1 \alpha$ expression under hypoxic conditions and promoted cell apoptosis. Overall, these results revealed that combining the EGR1 promoter and ING4 ORF using a lentivirus system may be a promising therapeutic strategy with which to enhance radiosensitivity controlled by
\end{abstract}

Correspondence to: Dr Hao-Ping Xu, Department of Radiation Oncology, Ruijin Hospital, Shanghai Jiaotong University School of Medicine, 197 Ruijin Er Road, Shanghai 200025, P.R. China

E-mail: xhp11701@rjh.com.cn

Key words: HeLa, human early growth response factor-1, human inhibitor of growth 4 , radiation therapy radiation. However, further studies using in vivo models are required to confirm these findings.

\section{Introduction}

Cervical cancer is the fourth most common type of cancer among women worldwide, and the second most common in low- and middle-income countries according to the data from GLOBOCAN 2018, with $>85 \%$ of new cases occurring in developing countries $(1,2)$. Radiotherapy is widely used, particularly for locally advanced cervical cancer (3). Following cell cycle arrest, ionizing radiation-induced apoptosis will occur if DNA damage is not repaired (4). Strategies to enhance the expression levels of pro-apoptotic genes could be applied in gene-radiotherapy (5). Recently, gene-radiotherapy, which combines gene therapy with radiotherapy, has shown promising effects (6).

It has been demonstrated that the tumor suppressor inhibitor of growth 4 (ING4) is deleted in numerous types of cancer (7). ING4 serves an important role in cell proliferation, apoptosis, cell cycle arrest, migration and vascularization, and these are pivotal to tumor progression (7-11). The radiation-inducible early growth response 1 (EGR1) promoter, which includes six serum response elements sensitive to ionizing radiation $(12,13)$, has attracted particular attentin. Previous studies have indicated that the EGR1 promoter can enhance the expression of its downstream genes, such as TNF- $\alpha$ and IFN- $\gamma(14,15)$.

During the process of tumor development, cells in hypoxia account for $10-50 \%$ of the tumor environment (16). Hypoxic tumor cells cause resistance to radiotherapy and chemotherapy, leading to tumor recurrence and distant metastasis (17). Hypoxia serves a vital role in angiogenesis (18). Furthermore, the hypoxia response element, upstream of the EGR1 promoter, can enhance the radiation-induced upregulation of therapeutic genes (19).

The present study combined the EGR1 promoter and ING4 open reading frame (ORF) as a cassette, which was integrated into HeLa cells to develop a cell line. Subsequently, the effects of ING4 on cell cycle arrest and cell proliferation were investigated when ING4 was induced using various doses of irradiation. Furthermore, the function of ING4 in hypoxia 
in HeLa cells was examined. Radiation and gene-combined treatment, termed gene-radiotherapy, exhibited a more prominent effect in HeLa cervical cancer cells in vitro.

\section{Materials and methods}

Cell culture and transfection. The human cervical carcinoma HeLa cell line was purchased from The Cell Bank of Type Culture Collection of Chinese Academy of Sciences and maintained at $37^{\circ} \mathrm{C}$ in DMEM (HyClone; Cytiva) supplemented with 10\% FBS (Gibco; Thermo Fisher Scientific, Inc.) and $100 \mathrm{U}$ penicillin-streptomycin. The cells were grown in a humidified atmosphere of $5 \% \mathrm{CO}_{2}$ and $95 \%$ air.

$\mathrm{HeLa}$ cells in the exponential phase of growth were treated with trypsin at $37^{\circ} \mathrm{C}$ for 1 minute. After terminating digestion with $10 \%$ FBS, the cells were resuspended in complete culture medium. A total of $2 \times 10^{5}$ cells were inoculated into each well of a 6-well plate and allowed to grow for $24 \mathrm{~h}$ prior to transfection. Subsequently, the cells in each well were transiently transfected with $2 \mu \mathrm{g}$ p-enhanced green fluorescent protein (EGFP)-N1 (Sigma-Aldrich; Merck $\mathrm{KGaA}$ ) or pEGR1-EGFP-N1 at room temperature (pEGR1-N1 was kindly provided by Dr Gerald Thiel from University of Saarland Medical Center, D-66421 Homburg, Germany) using $8.0 \mu 1$ Lipofectamine 2000 (Invitrogen; Thermo Fisher Scientific, Inc.). The transfection mixture was replaced with fresh culture medium at $6 \mathrm{~h}$ after transfection and was irradiated at $24 \mathrm{~h}$. The fluorescence images were captured at $72 \mathrm{~h}$ post-transfection using a fluorescence microscope (Nikon 80i; Nikon Corporation).

Construction of vectors. The mammalian cellular expression vector, pEGFP-N1 (6085-1), was purchased from Addgene, Inc. The CMV IE promoter was removed by endonucleases AseI and EcoRI. The promoter of human EGR1 ranging between -792 and 268 was isolated using primer pairs pEGR1-AseI-F/pEGR1-EcoRI-R and then directly cloned into the pEGFP-N1 vector, which was designated as pEGR1-EGFP-N1.

Similarly, the CMV promoter from construct pLV-mCherry(2A)puro (VL3405; Inovogen Tech. Co.) was replaced by the EGR 1 promoter using primer pairs pEGR1-ClaI-F/pEGR1-EcoRI-R and then used to develop the pEGR1-LV-mCherry(2A)puro vector. Furthermore, the human ING4 was cloned into pEGR1-LV-mCherry(2A)puro by EcoRI and XhoI with the hING4-EcoRI-F/hING4-XhoI-R primerpairs and the new construct was named pEGR1-LV-mCherry(2A) puro-hING4. Additionally, LV-mCherry(2A)puro-hING4 without any promoter was prepared.

Virus and cell lines. The recombinant lentivirus was obtained from HEK293T cells (Cell Bank of the Chinese Academy of Science) by transient transfection of lentivirus construct as well as helper plasmids psPAX2 (Addgene; cat. no. 12259) and MD2.G (Addgene; cat. no. 12260). Briefly, $1.0 \times 10^{6} 293 \mathrm{~T}$ cells were plated in a $6-\mathrm{cm}$ dish and cultured for 18-24 h. The cells were then co-transfected with $1.7 \mu \mathrm{g}$ pLV-mCherry(2A)puro, pEGR1-LV-mCherry(2A)puro, pEGR1-LV-mCherry(2A)puro-hING4 or LV-mCherry(2A) puro-hING4, $1.13 \mu \mathrm{g}$ psPAX2 and $0.57 \mu \mathrm{g}$ pMD2.G using
Lipofectamine 2000 (Invitrogen; Thermo Fisher Scientific, Inc.). 293T cells were maintained in DMEM medium with high glucose (Hyclone, cat. no. SH30243.01) supplemented with $10 \%$ fetal bovine serum (Gibco; Thermo Fisher Scientific, Inc; cat. no. 10099141 ) at $37^{\circ} \mathrm{C}$ in $5 \% \mathrm{CO}_{2}$. The supernatant containing the lentivirus was harvested at 48 and $72 \mathrm{~h}$ and then filtered through a $0.45-\mu \mathrm{m}$ low protein-binding polysulfonic filter (EMD Millipore). HeLa cells were inoculated in a 6 -well plate in advance at a density of $1.5 \times 10^{5}$ cells per well and presented with $\sim 60 \%$ confluency following incubation for $20 \mathrm{~h}$. The cells were then infected with $1 \mathrm{ml}$ lentivirus suspension in the presence of $8 \mu \mathrm{g} / \mathrm{ml}$ polybrene (Chemicon International; Thermo Fisher Scientific, Inc.). Following transduction for $48 \mathrm{~h}, \mathrm{HeLa}$ cells were selected with $2.0 \mu \mathrm{g} / \mathrm{ml}$ puromycin for 10 days until all blank control cells were dead. The puromycin-resistant cells were stably infected with exogenous genes.

Cell cycle analysis. HeLa cells stably integrated with LV-mCherry(2A)puro-hING4 or pEGR1-LV-mCherry(2A) puro-hING4 were inoculated in a 6-well plate and were allowed to grow for $24 \mathrm{~h}$. The cells were then exposed to X-rays with a dose ranging between 0 and 8 Gy ( 2 Gy gradient). Following growth for a further $48 \mathrm{~h}$, the cells were treated with trypsin at $37^{\circ} \mathrm{C}$ for $1 \mathrm{~min}$ and subjected to centrifugation at $800 \mathrm{x} \mathrm{g}$ for $5 \mathrm{~min}$ at $4^{\circ} \mathrm{C}$. After rinsing with $1 \mathrm{X}$ PBS, the cells were fixed in cold $\left(4^{\circ} \mathrm{C}\right) 70 \%$ ethanol and stored at $-20^{\circ} \mathrm{C}$ overnight. Following RNA removal using $50 \mu \mathrm{g} / \mathrm{ml}$ RNaseA (cat. no. R4875; Sigma-Aldrich; Merck KGaA) for $30 \mathrm{~min}$ at $37^{\circ} \mathrm{C}$, cells were stained with $500 \mu \mathrm{g} / \mathrm{ml}$ propidium iodide (eBioscience ${ }^{\mathrm{TM}}$, Thermo Fisher Scientific, Inc.) at room temperature for $15 \mathrm{~min}$. Subsequently, the cells were subjected to fluorescence-activated cell sorting (FACS)-based cell cycle analysis using a flow cytometer (FACSCalibur; BD Immunocytometry Systems) with CellQuest software (version 5.1). The percentages of cells in the sub- $G_{1}$ phase and different cell cycle phases were used as an index to evaluate the levels of cell apoptosis and cell cycle distribution, respectively.

Western blot analysis. Cells were lysed in 2X SDS buffer consisting of $0.1 \mathrm{M}$ Tris. $\mathrm{Cl}, 0.2 \mathrm{M}$ DTT, 4\% SDS and $20 \%$ Glycerol and subsequently prepared for three cycles of boiling $\left(95-100^{\circ} \mathrm{C}, 5-10 \mathrm{~min}\right)$ and cooling $\left(4^{\circ} \mathrm{C}, 5-10 \mathrm{~min}\right)$. The proteins were quantified using the Bradford method and subsequently mixed with $0.2 \%$ Bromophenol blue. A total of $15 \mu \mathrm{g}$ protein was loaded into each lane. Whole cell lysates were subjected to SDS-PAGE for protein separation and then electrophoretically transferred to a nitrocellulose membrane (Axygen; Corning Inc.), followed by blocking using PBS containing 5\% fat-free milk. The nitrocellulose membranes were incubated with a rabbit polyclonal antibody against ING4 (cat. no. ab113425; Abcam), an antibody against hypoxia-inducible factor $1 \alpha$ (HIF-1 $\alpha$; cat. no. 14179; Cell Signaling Technology, Inc.) and a rabbit polyclonal antibody against $\beta$-actin (cat. no. 103030001; HarO, http://www.lifeqho.com/pd.jsp?id=6\#_jcp=2) overnight at $4^{\circ} \mathrm{C}$. Subsequently, the membranes were incubated with a HRP-conjugated rabbit IgG secondary antibody (cat. no. 7074; Cell Signaling Technology, Inc. https://www.cellsignal. 

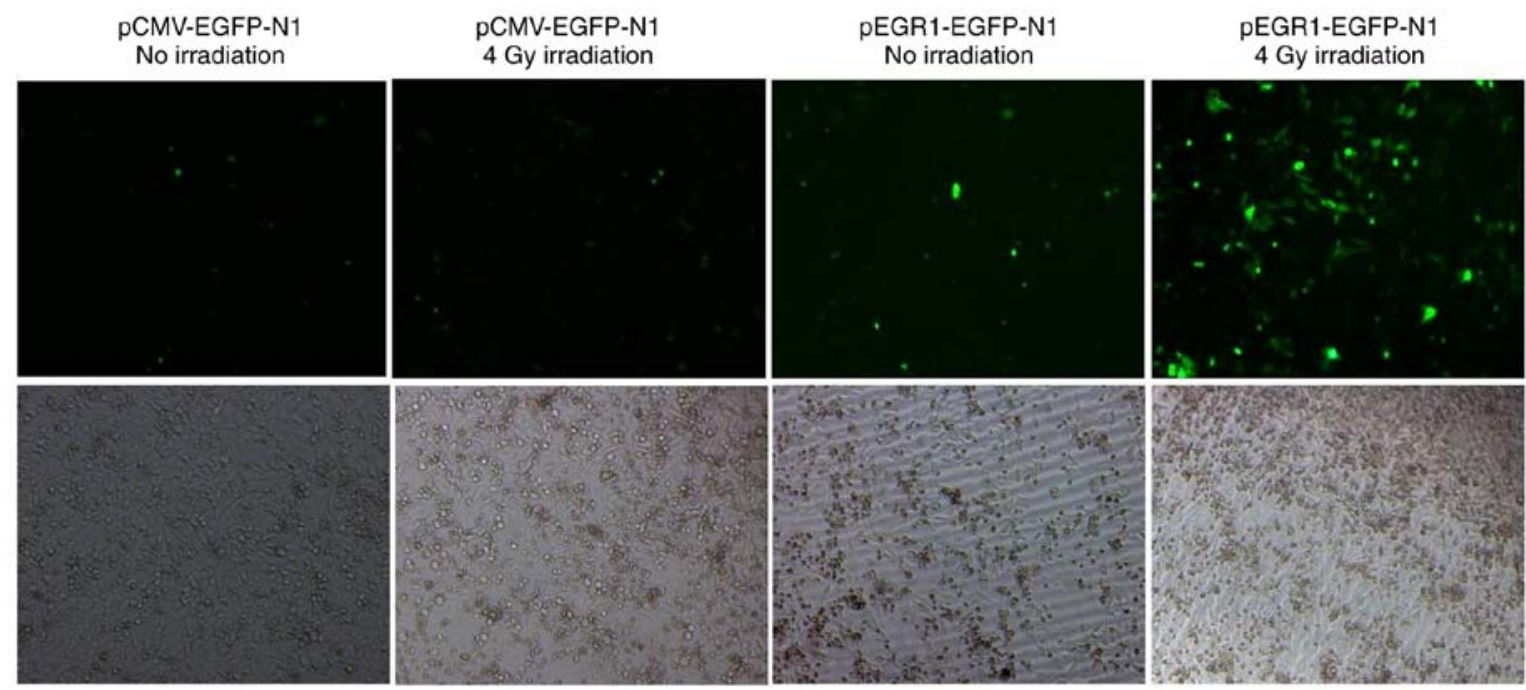

Figure 1. Irradiation-activated EGR1 promoter drives EGFP expression in transiently transfected HeLa cells. HeLa cells were transiently transfected with pEGFP-N1 or pEGR1-EGFP-N1 and exposed to irradiation of 4-Gy X-rays. In the upper panels, green fluorescence protein was observed, and its relative optical microscopy images were shown in the lower panels. Particularly, increased EGFP was visualized when the EGR1 promoter was exposed to X-rays (upper right panels). Magnification, x100. EGFP, enhanced green fluorescent protein; EGR1, early growth response factor-1.

com/products/secondary-antibodies/anti-rabbit-igg-hrp-linkedantibody/7074? $\mathrm{Ntk}=$ Products $\& \mathrm{Ntt}=7074$ ) for $1.5 \mathrm{~h}$ at room temperature. The immunolabeled proteins were detected using an immobilon western chemiluminescent HRP Substrate (WBKLS0500; MilliporeSigma ${ }^{\mathrm{TM}}$ ).

Anchorage-independent growth analysis. HeLa cells infected with LV-mCherry(2A)puro-hING4 or pEGR1-LV-mCherry(2A)puro-hING4 were trypsinized and suspended in culture medium. A total of 900 cells were seeded in each well of a 6-well plate with complete medium and were grown for $24 \mathrm{~h}$. The cells were then radiated by X-rays at a dose of 8,6,4,2 and $0 \mathrm{~Gy}$. When visible clones were observed for cells free from irradiation, all treated cells were subjected to fixation by $100 \%$ methanol at $4^{\circ} \mathrm{C}$ for $10 \mathrm{~min}$ and then stained for $1 \mathrm{~h}$ with $0.1 \%$ crystal violet at room temperature. After removing the dye, colonies containing $>50$ cells were counted (captured using a digital camera and the colonies in the photos were counted).

Cobalt chloride-imitated hypoxia. Cobalt chloride is an additive and widely used to imitate hypoxia in cell culture (20). The present study used cobalt chloride (cat. no. 232696; Sigma-Aldrich; Merck KGaA) at $100 \mu \mathrm{M}$ to treat the HeLa cells (at a density of $1.5 \times 10^{5} / \mathrm{ml}$ ), maintained at $37^{\circ} \mathrm{C}$ in a humidified atmosphere with $5 \% \mathrm{CO}_{2}$, following $\mathrm{X}$-ray exposure.

Statistical analysis. Statistical analysis was performed using SPSS 20.0 software (IBM Corp). All experiments were performed in triplicate wells for each condition and repeated at least twice. Data are presented as the mean \pm standard error. Error bars indicate the standard deviation. Generally, the one-way ANOVA method was used to evaluate the differences among treatments. Multiple comparisons among the groups were performed using the Bonferroni method. $\mathrm{P}<0.05$ was considered to indicate a statistically significant difference.

\section{Results}

Promoter of EGRl is sensitive to X-ray irradiation. The pEGFP-N1 or pEGR1-EGFP-N1 plasmid, in which the CMV promoter was replaced by the promoter of the EGR1 gene, was transfected into HeLa cells. At $24 \mathrm{~h}$ after transfection, cells were subjected to irradiation with X-rays at $4 \mathrm{~Gy}$ and fluorescence was observed at $48 \mathrm{~h}$ post-exposure to $\mathrm{X}$-rays. The results indicated that the CMV promoter did not respond to irradiation induction; however, the EGR1 promoter was sensitive to X-ray exposure (Figs. 1 and S1). Therefore, the EGR1 promoter was inducible by radiation and promoted the transcription and expression of its downstream gene.

HeLa cells stably integrated with the EGR1 promoter and the ING4 ORF cassette were screened and set up as a cell line. The cells expressed an intense red fluorescence protein, mCherry, following 4 Gy irradiation. By contrast, no red fluorescence was observed when the cells were not exposed to $\mathrm{X}$-rays (Fig. 2A).

The present study further examined whether the EGR1 promoter was sensitive to X-ray irradiation based on translation levels. HeLa cells with an EGR1-driven ING4 ORF were exposed to irradiation at the $0,2,4,6$ or $8 \mathrm{~Gy}$ for $48 \mathrm{~h}$, and proteins were then harvested and used to detect ING4 expression via western blot analysis. ING4 protein exhibited a dose-dependent expression pattern after the cells were irradiated with X-rays (Fig. 2C). However, HeLa cells integrated only with ING4 ORF did not exhibit enhanced ING4 protein expression following X-ray irradiation (Fig. 2B).

Therefore, it was concluded that the EGR1 promoter was sensitive to X-ray induction according to transient transfection-based green fluorescence protein, stably integration-based red fluorescence protein and ING4 protein expression. Since the purpose of the present study was to examine the responsiveness of the EGR1 promoter but not ING4 to irradiation, other negative controls, such as pEGR1-0, pCMV-ING4 and pING4 were not additionally evaluated. 
A
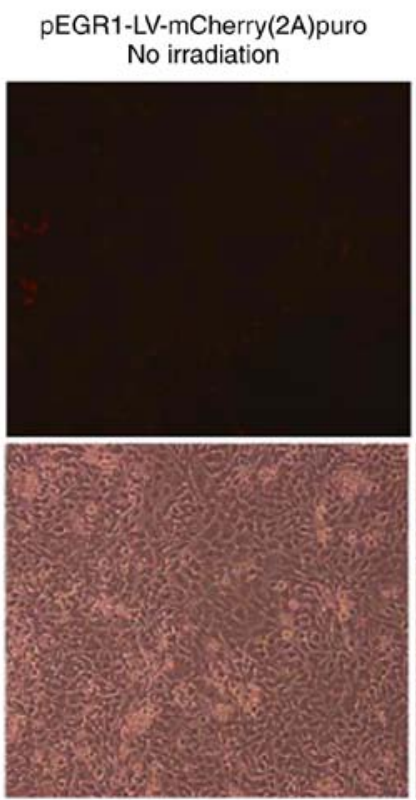

pEGR1-LV-mCherry(2A)puro

4 Gy irradiation
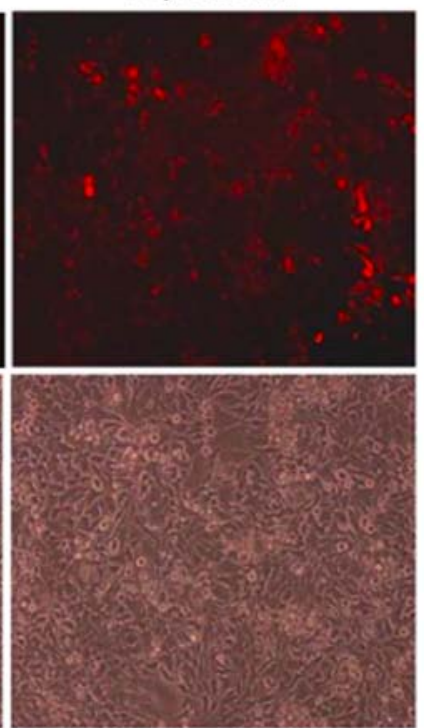

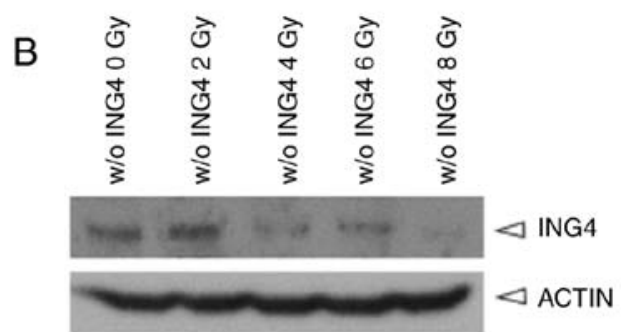

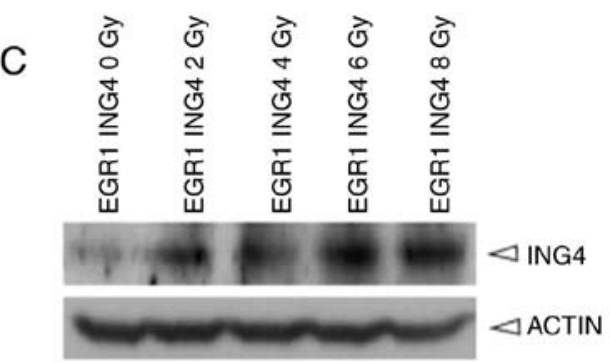

Figure 2. Irradiation-activated EGR1 promoter drives mCherry and ING4 expression in stably integrated HeLa cells. (A) HeLa cells were stably integrated with EGR1 promoter and mCherry ORF. mCherry expression increased due to the positively regulated promoter activity under irradiation (upper right panel). Magnification, x100. (B) ING4 expression was detected by western blotting. HeLa cells stably integrated with ING4 ORF without the EGR1 promoter irradiated by different doses of X-ray exhibited ING4 expression was hardly visible. (C) ING4 expression was monitored by western blotting. HeLa cells stably integrated with EGR1 promoter and ING4 ORF exhibited increased ING4 expression following treatment with a gradient of X-rays. EGR1, early growth response factor-1; ING4, inhibitor of growth 4; ORF, open reading frame.

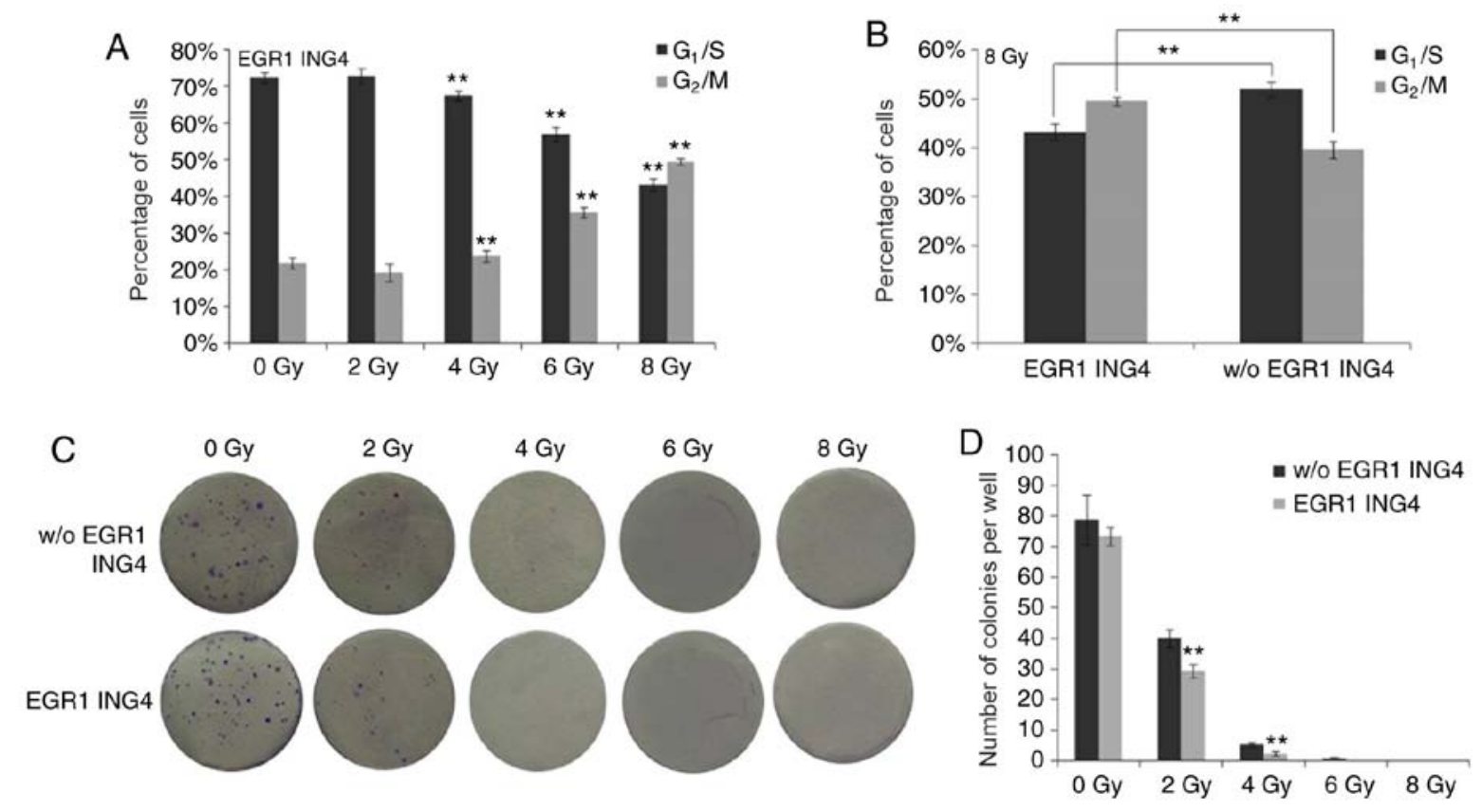

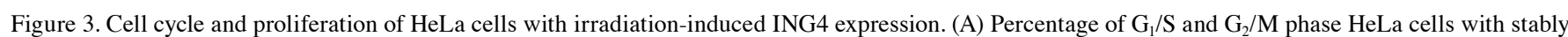
integrated EGR1 promoter and ING4 open reading frame irradiated by X-rays. Compared with those of HeLa cells without irradiation, higher percentages of cells in $\mathrm{G}_{2} / \mathrm{M}$ phase and lower percentages of cells in $\mathrm{G}_{1} / \mathrm{S}$ phase were observed in HeLa cells treated with 4,6 and 8 Gy irradiation. ${ }^{* *} \mathrm{P}<0.001$ vs. cell percentage without irradiation $(0 \mathrm{~Gy}$ ). (B) Distribution of phases of HeLa cells with or without inducible ING4 expression. ING4 expression significantly increased the percentage of cells in $\mathrm{G}_{2} / \mathrm{M}$ phase and decreased the percentage of cells in $\mathrm{G}_{1} / \mathrm{S}$ phase ${ }^{* *} \mathrm{P}=0.008$ vs. without EGR1 promoter. (C) The photo of colony formation assay of HeLa cells with and without inducible ING4 expression under irradiation. With increasing irradiation, decreased numbers of colonies were observed for both groups. (D) Comparison of the colony numbers between ING4-expressing HeLa cells and non-inducible cells. Once ING4 was induced, less colonies were observed in HeLa cells. ${ }^{* *} \mathrm{P}<0.001$ vs. cell number without irradiation ( $\left.0 \mathrm{~Gy}\right)$. EGR1, early growth response factor-1; ING4, inhibitor of growth 4.

ING4 induces $G_{2} / M$ phase arrest. With the increment of ING4 expression caused by the increasing doses of irradiation, the percentage of cells in the $\mathrm{G}_{2} / \mathrm{M}$ phase gradually increased along with a sequential decrease in the number of cells in the $\mathrm{G}_{1} / \mathrm{S}$ phase $(\mathrm{P}<0.001$; Figs. $3 \mathrm{~A}$ and 4$)$. In particular, the percentage of cells in the $\mathrm{G}_{2} / \mathrm{M}$ phase was statistically signifi- 

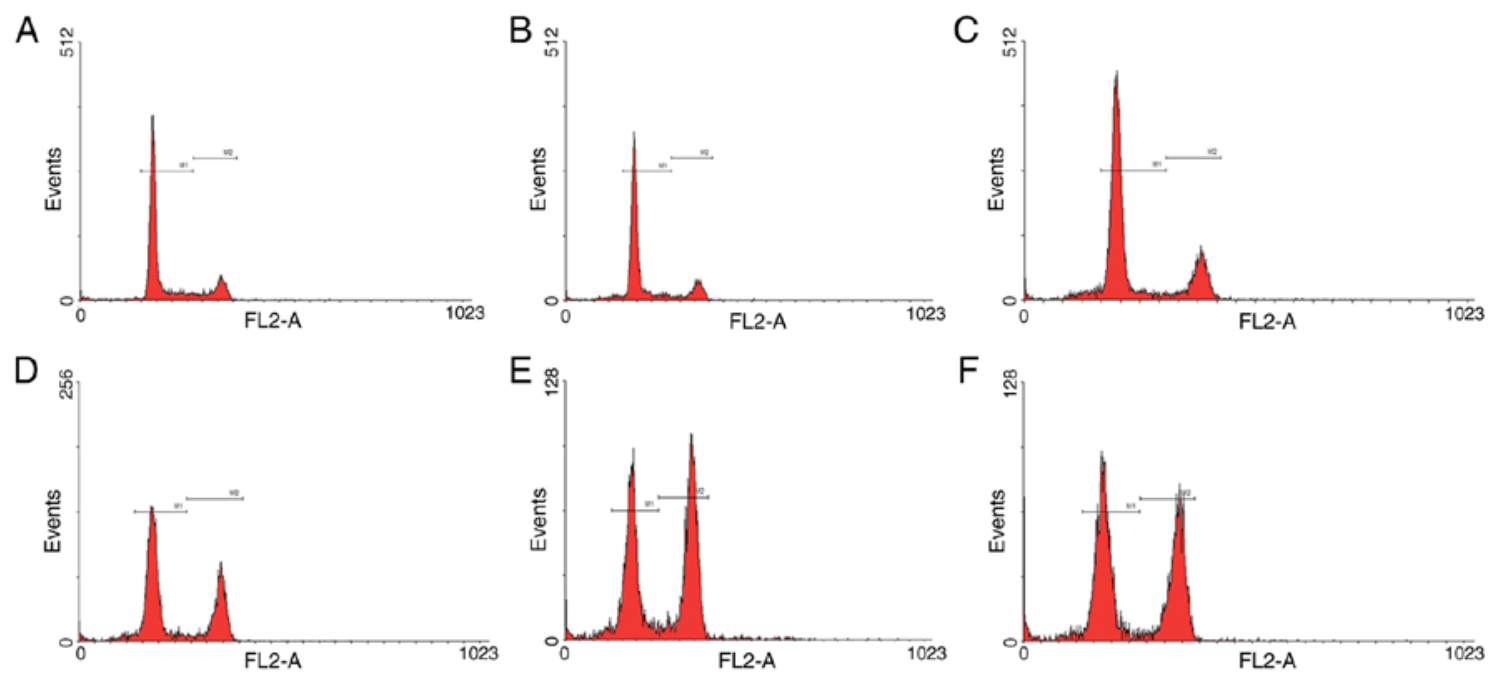

Figure 4. Cell cycle analyses of HeLa cells by flow cytometry under the control of different doses of irradiation. The cell counts of HeLa cells stably integrated with EGR1 promoter and ING4 ORF, irradiated by (A) 0, (B) 2, (C) 4, (D) 6 and (E) 8 Gy, in $\mathrm{G}_{1} / \mathrm{S}_{\text {and }} \mathrm{G}_{2} / \mathrm{M}$ phase. (F) The cell counts of HeLa cells stably integrated with ING4 ORF without irradiation-induced EGR1 promoter, and irradiated by $8 \mathrm{~Gy}$, in $\mathrm{G}_{1} / \mathrm{S}_{2}$ and $\mathrm{G}_{2} / \mathrm{M}$ phase. Events, cell counts; M1, $\mathrm{G}_{1} / \mathrm{S}$ phase; $\mathrm{M} 2, \mathrm{G}_{2} / \mathrm{M}$ phase; EGR1, early growth response factor-1; ING4, inhibitor of growth 4; ORF, open reading frame.

A

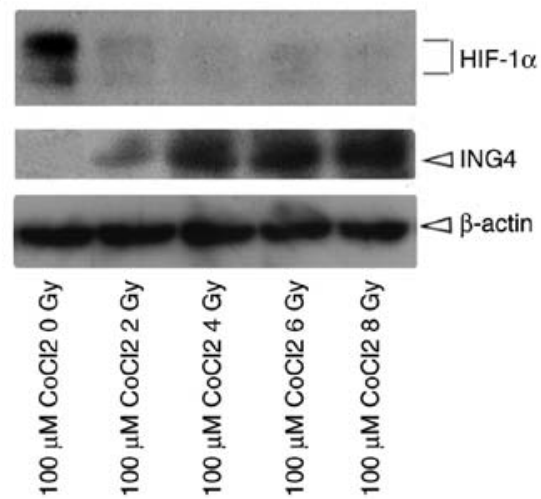

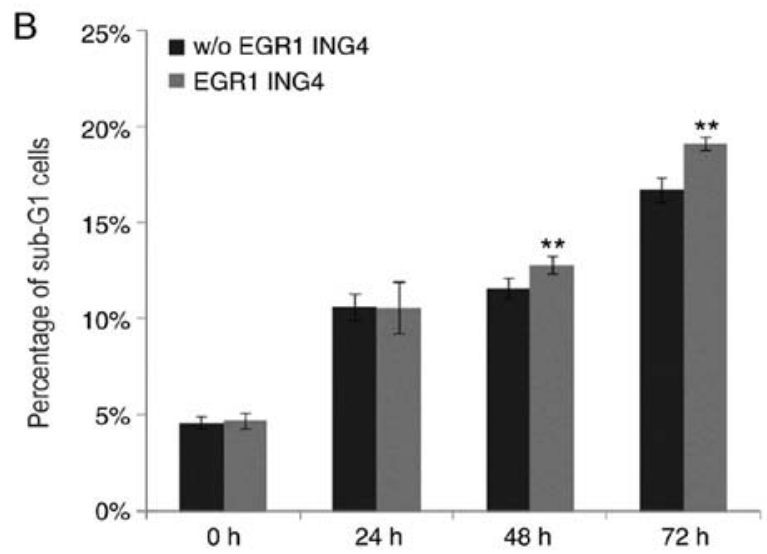

Figure 5. ING4 inhibits HIF-1 $\alpha$ expression and promotes cell apoptosis. (A) Inducible ING4 expression and its effect on HIF-1 $\alpha$ expression. When treated with CoCl2-imitated hypoxia, HeLa cells exhibited high expression levels of HIF-1 $\alpha$ which were inhibited by ING4 induced by irradiation. (B) Effect of ING4 on HeLa cell apoptosis in CoCl2-imitated hypoxia under 8 Gy irradiation. ING4 expression significantly promoted the apoptosis of HeLa cells at 48 and 72 h compared with cells transfected with 0 -ING4 (without EGR1 promoter), ${ }^{* *} \mathrm{P}<0.05$.. CoCl2, cobalt chloride; EGR1, early growth response factor-1; HIF-1 $\alpha$, hypoxia-inducible factor $1 \alpha$; ING4, inhibitor of growth 4.
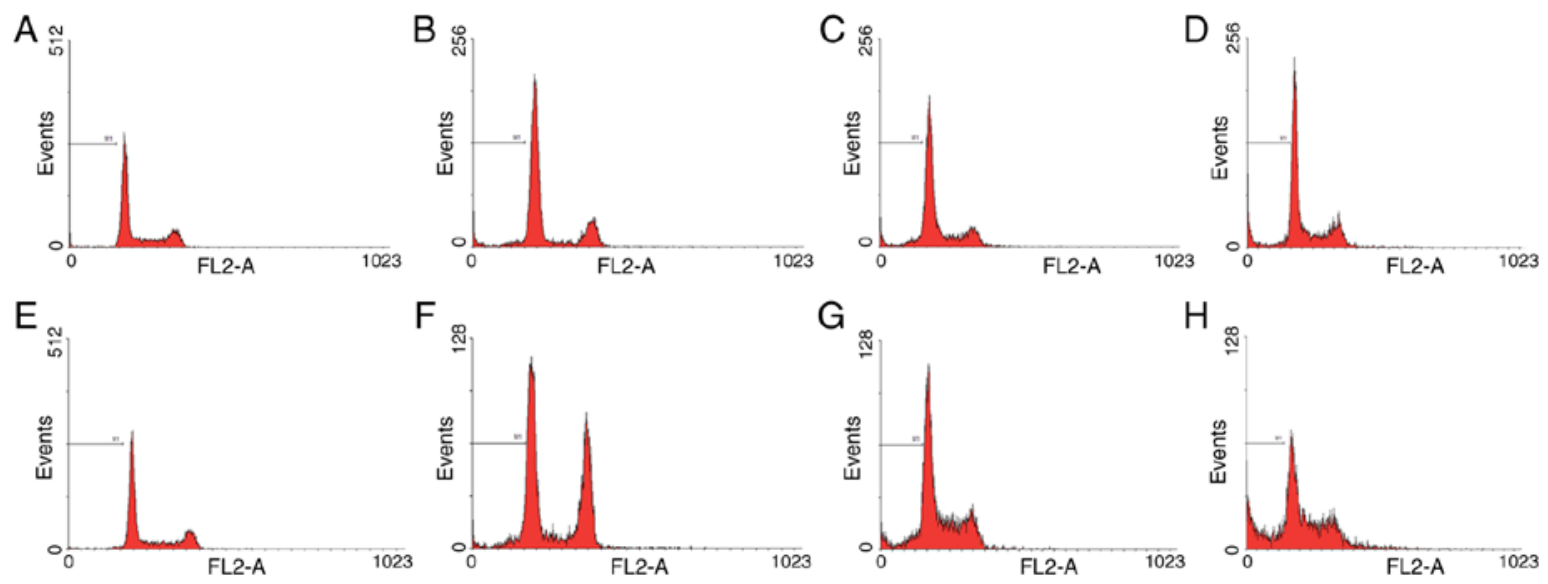

Figure 6. Apoptosis analysis of HeLa cells by flow cytometry with irradiation under hypoxia. Percentages of sub-G $\mathrm{G}_{1}$ phase HeLa cells stably integrated with ING4 ORF without irradiation-induced EGR1 promoter, irradiated and analyzed at (A) 0, (B) 24, (C) 48 and (D) $72 \mathrm{~h}$ post-irradiation. Percentages of sub- $\mathrm{G}_{1}$ phase HeLa cells stably integrated with EGR1 promoter and ING4 ORF, irradiated and analyzed at (E) 0, (F) 24, (G) 48 and (H) $72 \mathrm{~h}$ post-irradiation. $\mathrm{M} 1$, percentages of sub- $\mathrm{G}_{1}$ phase cells (apoptotic cells); EGR1, early growth response factor-1; ING4, inhibitor of growth 4; ORF, open reading frame. 
cantly higher in the ING4-expressing HeLa cells than in the HeLa cells without ING4 expression when 8 Gy irradiation was employed $(\mathrm{P}=0.005)$. By contrast, the number of cells in the $G_{1} / S$ phase was lower in the ING4-expressing HeLa cells than in the HeLa cells without ING4 expression $(\mathrm{P}=0.008$; Figs. 3B and 4). Therefore, irradiation-induced ING4 expression led to $G_{2} / M$ phase arrest, which may be responsible for the growth retardation (decreased number of the cells) of these HeLa cells (Fig. 3D).

ING4 inhibits the proliferation of HeLa cells. With the increase in the irradiation dose, ING4 expression was increased in HeLa cells, which resulted in less colonies being formed compared with in the HeLa cells not expressing ING4 (2 Gy, $\mathrm{P}=0.009$; $4 \mathrm{~Gy}, \mathrm{P}=0.003)$. By contrast, no statistically significant differences were observed in the number of colonies between the groups of HeLa cells not exposed to irradiation (0 Gy; Fig. 3C and D). No obvious colonies were observed following irradiation with $6 \mathrm{~Gy}$ or $8 \mathrm{~Gy}$.

ING4 inhibits HIF-1 $\alpha$ expression. In the present study, following irradiation with X-rays at various doses, HeLa cells were treated with $100 \mu \mathrm{M}$ cobalt chloride for 24,48 or $72 \mathrm{~h}$. Proteins were then collected from HeLa cells. HIF-1 $\alpha$ expression markedly decreased while ING4 expression increased (Fig. 5A).

ING4 promotes irradiation-induced apoptosis under hypoxic conditions. Furthermore, the proportions of HeLa cells in the sub- $\mathrm{G}_{1}$ phase under hypoxic conditions and following X-ray exposure were detected. At 48 and 72 h, ING4 increased the proportion of cells in the sub- $\mathrm{G}_{1}$ phase following 8 Gy irradiation, which indicated that a greater number of HeLa cells was apoptotic ( $\mathrm{P}=0.04$ and $\mathrm{P}=0.03$, respectively; Figs. 5B and 6). These results demonstrate that ING4 could promote cell apoptosis under hypoxic conditions.

\section{Discussion}

Hypoxic cancer cells are more resistant to irradiation compared with fully oxygenated cells (21). Therefore, the extent of tumor hypoxia is one of the most crucial biological factors affecting the outcomes of radiotherapy (22). Furthermore, HIF-1 has been identified to serve a pivotal role in hypoxia-mediated radioresistance (23). Research on the effects of hypoxia has increased in recent years; however, limited progress has been achieved in previous studies. On the other hand, dose escalation that aims to increase tumor control may observed the adverse effects of the normal tissues nearby. Therefore, enhancing the radiosensitivity of tumor cells, including overcoming hypoxia, is considered critical in order to achieve successful radiation therapy (24).

The strategies used to enhance radiosensitivity in the present study included three parts. At first, ING4 suppressed HIF-1 expression, which exerts a promoting effect on tumor growth under hypoxic conditions (21). Secondly, ING4 induced $\mathrm{G}_{2} / \mathrm{M}$ phase arrest and the apoptosis of HeLa cells. Several previous studies have reported similar results (25-28). The third part is that a lentiviral gene therapy vector was constructed, containing human ING4 and its upstream promoter, EGR1, which share the radiation-inducible characteristics to activate the transcription of downstream genes. When the experimental model was exposed to external irradiation, ING4 was activated by the EGR1 promoter and targeted to be expressed in irradiated sites. Therefore, the irradiation-sensitive promoter, EGR1, facilitated therapeutic gene expression under the control of ionizing radiation. Thus, the promoting effect on tumor growth by hypoxic cells was inhibited when HIF-1 was suppressed by ING4. Combined with the role of ING4 in regulating the cell cycle, synergetic radiosensitizing effects were achieved simultaneously with radiation therapy. The findings of the present study may provide novel strategies for the application and efficacy evaluation of radiosensitization in cervical cancer.

Increasing evidence has indicated that ING4 serves an important role in cancer progression as a tumor suppressor (5). In lung cancer tissues, decreased ING4 expression is present in $~ 50 \%$ of cases and is associated with lymph node metastasis (29). Together with the results of other surveys, ING4 is a promising target in gene-radiotherapy (30-32).

Radiotherapy combined with tumor suppressor genes is increasingly being used in tumor therapy (5). To achieve combined therapy, the genetic modification of tumor cells is critical. In the present study, ING4, as a tumor suppressor, was introduced into HeLa cells by lentiviral transfection under the control of the EGR1 promoter. The EGR1 promoter is sensitive to radiation. Therefore, the combination of ING4 and radiation was more effective in the cellular model of human cervical cancer treatment. The findings of the present study may provide promising strategies for use in the treatment of other types of cancer. However, these findings warrant further investigation using in vivo models.

\section{Acknowledgements}

Not applicable.

\section{Funding}

The present study was supported by Shanghai Pujiang Program (grant no. 2019PJD029)

\section{Availability of data and materials}

The datasets used and/or analyzed during the present study are available from the corresponding author upon reasonable request.

\section{Authors' contributions}

HPX and YNJ were involved in design of the work. TM was involved in acquisition of data and drafting the initial manuscript. HPX, XW and YNJ were the major contributors in revising the manuscript for important intellectual content. TM and HPX gave final approval of the version to be published. TM, XW, RG, WTS and MZ performed experimental research and analyzed experimental data. All authors have read and approved the final manuscript.

\section{Ethics approval and consent to participate}

Not applicable. 


\section{Patient consent for publication}

Not applicable.

\section{Competing interests}

The authors declare that they have no competing interests.

\section{References}

1. Bray F, Ferlay J, Soerjomataram I, Siegel RL, Torre LA and Jemal A: Global cancer statistics 2018: GLOBOCAN estimates of incidence and mortality worldwide for 36 cancers in 185 countries. CA Cancer J Clin 68: 394-424, 2018.

2. Bhatla N, Berek JS, Fredes MC, Denny LA, Grenman S, Karunaratne K, Kehoe ST, Konishi I, Olawaiye AB, Prat J, et al Revised FIGO staging for carcinoma of the cervix uteri. Int J Gynaecol Obstet 145: 129-135. 2019.

3. Chino J, Annunziata CM, Beriwal S, Bradfield L, Erickson BA, Fields EC, Fitch K, Harkenrider MM, Holschneider $\mathrm{CH}$, Kamrava M, et al: Radiation therapy for cervical cancer: Executive summary of an ASTRO clinical practice guideline. Pract Radiat Oncol 10: 220-234, 2020.

4. Lukas J, Lukas C and Bartek J: Mammalian cell cycle checkpoints: Signalling pathways and their organization in space and time. DNA Repair (Amst) 3: 997-1007, 2004

5. Zhao Y, Li Z, Sheng W, Miao J and Yang J: Radiosensitivity by ING4-IL-24 bicistronic adenovirus-mediated gene cotransfer on human breast cancer cells. Cancer Gene Ther 20: 38-45, 2013.

6. Jiang X, Zhang QL, Tian YH, Huang JC and Ma GL: RNA interference-mediated gene silencing of cyclophilin A enhances the radiosensitivity of PAa human lung adenocarcinoma cells in vitro. Oncol Lett 13: 1619-1624, 2017.

7. Liu Y, Yu L, Wang Y, Zhang Y, Wang Y and Zhang G: Expression of tumor suppressor gene ING4 in ovarian carcinoma is correlated with microvessel density. J Cancer Res Clin Oncol 138: 647-655, 2012.

8. Gunduz M, Nagatsuka H, Demircan K, Gunduz E, Cengiz B, Ouchida M, Tsujigiwa H, Yamachika E, Fukushima K, Beder L, et al: Frequent deletion and down-regulation of ING4, a candidate tumor suppressor gene at $12 \mathrm{p} 13$, in head and neck squamous cell carcinomas. Gene 356: 109-117, 2005.

9. Coles AH and Jones SN: The ING gene family in the regulation of cell growth and tumorigenesis. J Cell Physiol 218: 45-57, 2009.

10. Xu M, Xie Y, Sheng W, Miao J and Yang J: Adenovirus-mediated ING4 gene transfer in osteosarcoma suppresses tumor growth via induction of apoptosis and inhibition of tumor angiogenesis. Technol Cancer Res Treat 14: 369-378, 2015.

11. Yan R, He L, Li Z, Han X, Liang J, Si W, Chen Z, Li L, Xie G, $\mathrm{Li} \mathrm{W}$, et al: $\mathrm{SCF}(\mathrm{JFK})$ is a bona fide $\mathrm{E} 3$ ligase for ING4 and a potent promoter of the angiogenesis and metastasis of breast cancer. Genes Dev 29: 672-685, 2015.

12. Weichselbaum RR and Kufe D: Translation of the radio- and chemo-inducible TNFerade vector to the treatment of human cancers. Cancer Gene Ther 16: 609-619, 2009.

13. Kufe D and Weichselbaum R: Radiation therapy: Activation for gene transcription and the development of genetic radiotherapy-therapeutic strategies in oncology. Cancer Biol Ther 2 : 326-329, 2003

14. Liu LL, Smith MJ, Sun BS, Wang GJ, Redmond HP and Wang JH: Combined IFN-gamma-endostatin gene therapy and radiotherapy attenuates primary breast tumor growth and lung metastases via enhanced CTL and NK cell activation and attenuated tumor angiogenesis in a murine model. Ann Surg Oncol 16: 1403-1411, 2009

15. Yang W and Li XY: Anti-tumor effect of pEgr-interferon-gam ma-endostatin gene-radiotherapy in mice bearing Lewis lung carcinoma and its mechanism. Chin Med J (Engl) 118: 296-301, 2005
16. Arai M, Kawachi T, Setiawan A and Kobayashi $M$ Hypoxia-selective growth inhibition of cancer cells by furospinosulin-1, a furanosesterterpene isolated from an Indonesian marine sponge. ChemMedChem 5: 1919-26, 2010.

17. Toma-Daşu I, Daşu A and Karlsson M: The relationship between temporal variation of hypoxia, polarographic measurements and predictions of tumour response to radiation. Phys Med Biol 49: 4463-4475, 2004

18. Hickey MM and Simon MC: Regulation of angiogenesis by hypoxia and hypoxia-inducible factors. Curr Top Dev Biol 76: 217-257, 2006.

19. Leskov KS, Criswell T, Antonio S, Li J, Yang CR, Kinsella TJ and Boothman DA: When X-ray-inducible proteins meet DNA double strand break repair. Semin Radiat Oncol 11: 352-372, 2001.

20. Rovetta F, Stacchiotti A, Faggi F, Catalani S, Apostoli P, Fanzani A and Aleo MF: Cobalt triggers necrotic cell death and atrophy in skeletal C2C12 myotubes. Toxicol Appl Pharmacol 271: 196-205, 2013.

21. Rockwell S, Dobrucki IT, Kim EY, Marrison ST and Vu VT: Hypoxia and radiation therapy: Past history, ongoing research, and future promise. Curr Mol Med 9: 442-458, 2009.

22. Begg AC, Stewart FA and Vens C: Strategies to improve radiotherapy with targeted drugs. Nat Rev Cancer 11: 239-253, 2011

23. Moeller BJ and Dewhirst MW: HIF-1 and tumour radiosensitivity. Br J Cancer 95(1): 1-5. 2006 (Epub ahead of print).

24. Kaanders JH, Bussink J and van der Kogel AJ: ARCON: A novel biology-based approach in radiotherapy. Lancet Oncol 3: 728-737, 2002.

25. Li Z, Xie Y, Sheng W, Miao J, Xiang J and Yang J: Tumor-suppressive effect of adenovirus-mediated inhibitor of growth 4 gene transfer in breast carcinoma cells in vitro and in vivo. Cancer Biother Radiopharm 25: 427-437, 2010.

26. Du Y, Cheng Y and Su G: The essential role of tumor suppressor gene ING4 in various human cancers and non-neoplastic disorders. Biosci Rep 39: BSR20180773, 2019.

27. Xie Y, Zhang H, Sheng W, Xiang J, Ye Z and Yang J: Adenovirus-mediated ING4 expression suppresses lung carcinoma cell growth via induction of cell cycle alteration and apoptosis and inhibition of tumor invasion and angiogenesis. Cancer Lett 271: 105-116, 2008.

28. You Q, Wang XS, Fu SB and Jin XM: Downregulated expression of inhibitor of growth 4 (ING4) in advanced colorectal cancers: A non-randomized experimental study. Pathol Oncol Res 17: 473-477, 2011.

29. Wang QS, Li M, Zhang LY, Jin Y, Tong DD, Yu Y, Bai J, Huang Q, Liu FL, Liu A, et al: Down-regulation of ING4 is associated with initiation and progression of lung cancer. Histopathology 57: 271-281, 2010.

30. Galal El-Shemi A, Mohammed Ashshi A, Oh E, Jung BK, Basalamah M, Alsaegh A and Yun CO: Efficacy of combining ING4 and TRAIL genes in cancer-targeting gene virotherapy strategy: First evidence in preclinical hepatocellular carcinoma. Gene Ther 25: 54-65, 2018

31. Zhang H, Zhou X, Xu C, Yang J, Xiang J, Tao M and Xie Y: Synergistic tumor suppression by adenovirus-mediated ING4/PTEN double gene therapy for gastric cancer. Cancer Gene Ther 23: 13-23, 2016.

32. Wang Y, Yang J, Sheng W, Xie Y and Liu J: Adenovirus-mediated ING4/PTEN double tumor suppressor gene co-transfer modified by RGD enhances antitumor activity in human nasopharyngeal carcinoma cells. Int J Oncol 46: 1295-1303, 2015.

(i) $(-)$ This work is licensed under a Creative Commons Attribution-NonCommercial-NoDerivatives 4.0 International (CC BY-NC-ND 4.0) License. 